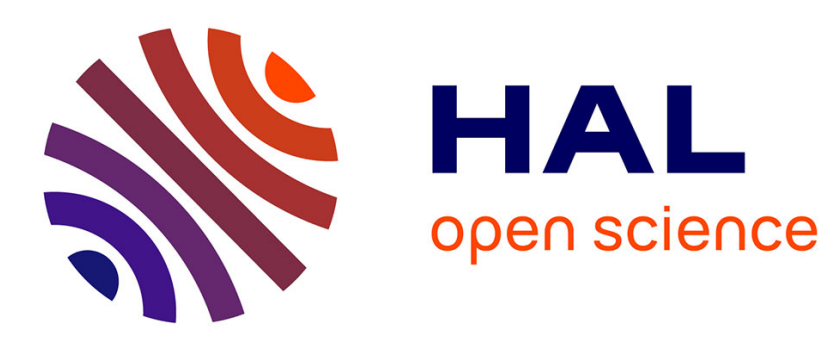

\title{
Dimension reduction for large-scale networked systems
}

\author{
Irinel-Constantin Morarescu, Romain Postoyan
}

\section{To cite this version:}

Irinel-Constantin Morarescu, Romain Postoyan. Dimension reduction for large-scale networked systems. 51st IEEE Conference on Decision and Control, CDC 2012, Dec 2012, Maui, Hawaii, United States. pp.CDROM. hal-00733802

\section{HAL Id: hal-00733802 \\ https://hal.science/hal-00733802}

Submitted on 19 Sep 2012

HAL is a multi-disciplinary open access archive for the deposit and dissemination of scientific research documents, whether they are published or not. The documents may come from teaching and research institutions in France or abroad, or from public or private research centers.
L'archive ouverte pluridisciplinaire HAL, est destinée au dépôt et à la diffusion de documents scientifiques de niveau recherche, publiés ou non, émanant des établissements d'enseignement et de recherche français ou étrangers, des laboratoires publics ou privés. 


\title{
Dimension reduction for large-scale networked systems
}

\author{
Irinel-Constantin Morărescu and Romain Postoyan *
}

September 19, 2012

\begin{abstract}
A methodology is proposed to approximate large-scale networked systems by a lower dimensional networked system. We first group the nodes into $m$ communities which will form the $m$ vertices of the reduced network. We then associate an appropriate scalar dynamics to each community; in that way, the dimension of the new model is equal to $m$. The main idea is to approximate each node trajectory by the trajectory of its community. The edges are derived by considering some linear combinations of the link strengths between the elements of each community. Finally, the initial conditions are selected to guarantee the asymptotic consistency of the reduced model with the original system. Thus, we prove the asymptotic convergence of any state of the original system to its corresponding community state according to some distance. It has to be emphasized that our approach is flexible as the user is free to select the reduced system dimension $m$.
\end{abstract}

\section{Introduction}

For any proof or the complete version of the paper please contact one of the authors

Networks and networked systems are ubiquitous in diverse areas of science and engineering, e.g. in biology, in communication systems, in economy etc. In the past decades, the analysis of dynamical networks has received an increasing interest in the control and dynamical systems communities. As a result, a distinct research field has appeared at the intersection of systems theory and graph theory, see $[1,2,3,4]$ to mention a few. A way to overcome the difficulties induced by the high dimension of these systems consists in developing approximate low dimensional models. Such approximations are of great interest for the simulation and the analysis of large-scale networked systems. Various

\footnotetext{
*Irinel-Constantin Morărescu and Romain Postoyan are with the Université de Lorraine, CRAN, UMR 7039 and the CNRS, CRAN, UMR 7039, France, constantin.morarescu@univ-lorraine.fr, romain.postoyan@univ-lorraine.fr
} 
methodologies are available for reducing the dimension and / or the complexity of linear and nonlinear systems (see $[5,6]$ and the references therein). When dealing with systems in network, we aim at working with a reduced model which allows us to approximate the trajectory of each node (not only some given outputs), which make the results in $[5,6]$ not suitable. In $[7,8]$, the nodes which are assumed to be synchronized are assimilated to a single node, giving rise to the concept of meta-populations. It has to be noted that the idea of grouping nodes is related to the problem of community or cluster detection in static networks, see for instance $[9,10,11,12]$. For networks of oscillators which are known to exhibit synchronization properties, reduced models have been developed to maintain the convergence speed and some asymptotic behaviour properties (see [13] and the references therein).

In this paper, we propose a methodology to approximate a class of linear networked systems by lower dimensional networked systems. We start by partitioning the nodes into $m$ groups called communities to which we assign a scalar state variable. The main idea is to approximate the trajectory of each node by the trajectory of its associated community. These communities form the nodes of the reduced network which is thus of dimension $m$. Their choice as well as their number can be freely set by the user. We define the community dynamics to be some projection of its inner element dynamics and we give a condition to select the initial condition of the reduced model. In that way, we show that the error between the community trajectory and the trajectory of its inner elements is upper bounded by a term that tends to zero as time grows according to some distance. The derived results are applied to the case where the collective dynamics are given by a stochastic matrix which ensures a consensus among all the nodes [14]. Like in [13], the approximation maintains the asymptotic behaviour of the original system but, in addition, we are also able to quantify the quality of the approximation at any time (even during the transient period). Furthermore, our approach is flexible as the dimension of the reduced model can be freely set depending on the desired level of accuracy. It has to be noted that we do not require the synchronization of all the nodes within a community as opposed to $[7,8]$.

The paper is organized as follows. After having recalled some definitions in Section 2, the problem we solve is presented in Section 3. The reduced model is built up in Section 4 and it is shown to appropriately approximate the original system in Section 5. We then focus on the case where the overall system dynamics is given by a stochastic matrix in Section 6 for which some of the previous assumptions are relaxed. An illustrative example is finally presented in Section 7.

\section{Preliminaries}

Let $G=(\mathcal{V}, \mathcal{E})$ denotes a connected directed graph with the set of vertices (nodes) $\mathcal{V}$ and the set of edges $\mathcal{E}$. Each node is labeled by $v_{i} \in \mathcal{V}, i=1, \ldots, n$ and one says that $(i, j) \in \mathcal{E}$ if there exists an edge between $v_{i}$ and $v_{j}$. 
Definition $1 A$ path in a given graph $G=(\mathcal{V}, \mathcal{E})$ is a union of edges $\bigcup_{k=1}^{p}\left(i_{k}, j_{k}\right)$ such that $i_{k+1}=j_{k}, \forall k \in\{1, \ldots, p-1\}, p \in \mathbb{N}$. Two nodes $i, j$ are connected in a graph $G=(\mathcal{V}, \mathcal{E})$ if there exists at least a path in $G$ joining $i$ and $j$ (i.e. $i_{1}=i$ and $j_{p}=j$ ). If all the nodes are connected we say the graph is strongly connected or simply connected if additionally the graph is undirected. In this paper, a community is defined as a group of nodes with no specific property.

\section{Problem formulation}

We consider a directed fixed graph $G=(\mathcal{V}, \mathcal{E})$. Each vertex $v_{i}, i \in\{1, \ldots, n\}$, represents a dynamic agent and the state trajectory of the networked system is given by $x(\cdot)=\left(x_{1}(\cdot), x_{2}(\cdot), \ldots, x_{n}(\cdot)\right)^{\top} \in \mathbb{R}^{n}$ where $x_{i}(t)$ is a real value assigned to $v_{i}$ at the moment $t$. It is worth noting that $x_{i}$ denotes generically the state of the agent $v_{i}$ while $x_{i}(t)$ and $x_{i}(\cdot)$ represent the state value at time $t$ and the state trajectory of the agent $v_{i}$, respectively.

The initial conditions are given by $x_{i}(0)=x_{i}^{0}, \forall i=\overline{1, n}$. The agents update their state by making a linear combination of its own state and the state of its neighbors

$$
x_{i}(t+1)=\sum_{j=1}^{n} a_{i j} x_{j}(t), \quad \forall i \in\{1, \ldots, n\}, \forall t \geq 0,
$$

where $a_{i j} \neq 0$ if and only if $(i, j) \in \mathcal{E}$. The collective dynamics is described by the matrix $A=\left[a_{i j}\right]_{1 \leq i, j \leq n}$ :

$$
x(t+1)=A x(t), \quad \forall t \geq 0 .
$$

Model (2) can be used to represent the temperature dynamics in buildings having a large number of rooms for instance (see [15]). In this case, $x_{i}$ represents the temperature of the room $i$. The temperature transfer between neighboring rooms is described by the gain $a_{i j}$ while $a_{i i}$ models the decentralized feedback action in room $i$. The dimension reduction is here related to the limitations on the deployment of sensors in the rooms. Thus, one approximates the temperature of neighboring rooms by the temperature given by a single sensor $\tilde{x}_{C_{i}}$. The model may also represent the process of negotiation in a collectivity in order to take a common decision, where $x_{i}$ represents the opinion of agent $i$. At each iteration, an agent updates its opinion by making an weighted average of its own opinion and those of its neighbors. In this case, the dimension reduction is related to the presence of moderators who lead the discussions in their group, collect an averaged opinion $\tilde{x}_{C_{i}}$, and exchange it with the others moderators.

We assume that the following condition holds in Sections 4-5.

Assumption 1 The matrix $A$ is symmetric non-negative primitive matrix with the spectral radius $\rho(A)=1$.

The above assumption is imposed to assure some convergence properties for the collective dynamics (see the next section for details) and allows us to consider the case of symmetric stochastic matrices. 
To build the reduced model, we start by defining a set of communities $C_{i}$, $i \in\{1, \ldots, m\}$ to which we associate a scalar state $\tilde{x}_{C_{i}} \in \mathbb{R}$. We define the state vector of the communities by $\tilde{x}=\left(\tilde{x}_{C_{1}}, \ldots, \tilde{x}_{C_{m}}\right) \in \mathbb{R}^{m}$. The number of communities $m \in\{1, \ldots, n\}$ is a design parameter and the groups $C_{i}, i \in$ $1, \ldots, m$ are freely chosen by the designer. In practice, $m$ and $C_{i}, i \in\{1, \ldots, m\}$ are usually chosen such that connectivity or dynamical properties are stronger inside the group than outside. This allows us to built the graph $\tilde{G}=(\tilde{\mathcal{V}}, \tilde{\mathcal{E}})$ where

- the set of vertices is $\tilde{\mathcal{V}}=\left\{C_{1}, \ldots, C_{m}\right\}$.

- the set of edges is $\tilde{\mathcal{E}}$ is defined such that an edge between $C_{i}$ and $C_{j}$ exists if and only if at least an edge connecting a vertex of $C_{i}$ with a vertex of $C_{j}$ exists in $\mathcal{E}$.

We are interested in solving the problem below:

Problem 1 Find a communities state dynamics and an appropriate initial condition $\tilde{x}^{0}$ such that it exists a function $\varphi_{m}: \mathbb{R}_{+} \mapsto \mathbb{R}_{+}$which tends to 0 as time grows and it holds that $\| x(t)-K \tilde{x}(t)) \| \leq \varphi_{m}(t)$, where $K \in \mathbb{R}^{n \times m}$ is a designed matrix.

\section{Design of the lower dimensional model}

We first present the dynamics that are assigned to each community, then we show how the initial conditions of the new system need to be selected.

\subsection{Community dynamics}

Since the matrix $A$ satisfies Assumption 1, the Perron-Frobenius theorem (see Section 8.3 in [16]) guarantees that:

- 1 is a simple eigenvalue of $A$.

- The components $u_{i}$ of the Perron eigenvector $u$ of $A$ associated to 1 are all positive.

- $\lim _{t \rightarrow \infty} A^{t}=u u^{\top}$.

The vector $u$ plays a key role in the design of the reduced model. We denote by $\left\{e_{1}, \ldots, e_{n}\right\}$ the canonical basis of $\mathbb{R}^{n}$ and $u_{C_{i}}=\sum_{i \in C_{i}} u_{i} e_{i} \in \mathbb{R}^{n}$ we introduce the following matrices

$$
\begin{aligned}
& K=\left(\frac{u_{C_{1}}}{\left\|u_{C_{1}}\right\|}, \frac{u_{C_{2}}}{\left\|u_{C_{2}}\right\|}, \ldots, \frac{u_{C_{m}}}{\left\|u_{C_{m}}\right\|}\right) \in \mathbb{R}^{n \times m}, \\
& E=K K^{\top} \in \mathbb{R}^{n \times n} .
\end{aligned}
$$


Lemma 1 The following properties hold:

1. $E$ is a projector i.e. $E^{2}=E$ and $E^{\top}=E$. So $\|E\|=1$;

2. $E u_{C_{i}}=u_{C_{i}}, \forall t \geq 0, i \in 1, \ldots, m$ and $E u=u$.

The state dynamics of each community is defined by

$$
\tilde{x}_{i}(t+1)=\sum_{j=1}^{m} \tilde{a}_{i j} \tilde{x}_{j}(t), \quad \forall t \geq 0
$$

where $\tilde{a}_{i j}$ are linear combinations of the link strength between elements in $C_{i}$ and $C_{j}$ which are defined by

$$
\tilde{a}_{i j}=\frac{1}{\left\|u_{C_{i}}\right\|\left\|u_{C_{j}}\right\|} u_{C_{i}}^{\top} A u_{C_{j}}, \quad \forall i, j .
$$

It is noteworthy that no link connects $C_{i}$ and $C_{j}$ if no elements of $C_{i}$ is connected with an element in $C_{j}$. Considering $\tilde{A}=\left(\tilde{a}_{i j}\right)_{1 \leq i, j \leq m}$, the collective dynamics of the communities state is then given by

$$
\tilde{x}(t+1)=\tilde{A} \tilde{x}(t), \quad \forall t \geq 0 .
$$

It has to be noted that $\tilde{A}=K^{\top} A K \in \mathbb{R}^{m \times m}$. We also notice that $A$ symmetric implies $\tilde{A}$ symmetric. An interesting observation that will be used in the sequel reveals that $K^{\top} K=I d_{m}$.

\subsection{Community initial conditions}

We recall that $\tilde{x}^{0}$ stands for the initial condition for the communities dynamics (5). The following proposition gives a condition for selecting $\tilde{x}^{0}$ guaranteing the asymptotic consistency of the reduced model with the original one.

Proposition 1 Let $w \triangleq K^{\top} u$. If $w^{\top} \tilde{x}^{0}=u^{\top} x^{0} \triangleq x^{*}$, then one obtains $\lim _{t \rightarrow \infty}\|x(t)-K \tilde{x}(t)\|=0$. Moreover, $w$ is the left Perron eigenvector of $\tilde{A}$.

Following Proposition 1, we select $\tilde{x}^{0}$ such that $w^{\top} \tilde{x}^{0}=u^{\top} x^{0}$. Therefore, a computationally simple choice is $\tilde{x}^{0}=K^{\top} x^{0}$. If we want to minimize the initial error we may compute $\tilde{x}^{0}$ such that $\tilde{x}(0)=\operatorname{argmin}_{w^{\top} \tilde{x}=u^{\top} x^{0}}\|K \tilde{x}-x(0)\|$.

Remark 1 Like in [13], it is possible to relate the spectral properties of the reduced model and the original one. Precisely, the spectrum of the matrix $\tilde{A}$ can be characterized as follows. If $\lambda$ is an eigenvalue of EA associated to the left eigenvector $v_{\lambda}$ then one and only one of the following statements holds:

1. $K^{\top} v_{\lambda}=0$.

2. $\lambda$ is an eigenvalue of $\tilde{A}$ associated to the eigenvector $w_{\lambda}=K^{\top} v_{\lambda}$. Indeed, if If $K^{\top} v_{\lambda} \neq 0$ one has $w_{\lambda}^{\top} \tilde{A}=w_{\lambda}^{\top} K^{\top} A K=v_{\lambda}^{\top} E A K=\lambda v_{\lambda}^{\top} K=\lambda w_{\lambda}^{\top}$. 


\section{$5 \quad$ Trajectory approximation bounds}

We are now ready to define the function $\varphi_{m}(\cdot)$ that solves Problem 1 with the matrix $K$ defined in (3).

Theorem 1 Let $1=\lambda_{1}(A)>\left|\lambda_{2}(A)\right| \geq \ldots \geq\left|\lambda_{n}(A)\right| \geq 0$ be the eigenvalues of the matrix $A$ and $\tilde{x}^{0}$ be chosen as in Proposition 1. A solution to Problem 1 is given by

$$
\begin{aligned}
\varphi_{m}(t)= & \left|\lambda_{2}(A)\right|^{t}(\|K \tilde{x}(0)-x(0)\| \\
& \left.+t\left\|E-I d_{n}\right\|\left\|K\left(\tilde{x}(0)-x^{*} w\right)\right\|\right) .
\end{aligned}
$$

We note that the dependence of $\varphi_{m}(\cdot)$ on $m$ in (6) is hidden in the structure of $K$ and $w$. In the particular case of stochastic symmetric matrices studied in the next section, this dependence is evident, see (11).

Remark 2 1) From Lemma $1, E$ is a projector different to $I d_{n}$ when $m<n$ as we can easily check that $I d_{n}-E$ is also a projector so $\left\|E-I d_{n}\right\|=1$. Consequently, (6) may be simplified as $\varphi_{m}(t)=\left|\lambda_{2}(A)\right|^{t}(\|K \tilde{x}(0)-x(0)\|+t \| K(\tilde{x}(0)-$ $\left.\left.x^{*} w\right) \|\right)$.

2) Due to the presence of the second term in the right hand side of (6), the function $\varphi_{m}(\cdot)$ may be increasing during few iterations before starting an almost exponential decrease to 0 (as seen in an example in Section 7, see Figure 1).

3) As mentioned in Section 3, the dimension of the reduced model $m$ can be freely selected. We show in simulations in Section 7 that the approximation accuracy degrades as $m$ decreases. For $m=n$ (i.e. no complexity reduction is made) one has $K=I d_{n}=\tilde{K} \Rightarrow E=I d_{n}$ and $\tilde{x}(0)=x(0)$. Thus, (6) states that the two trajectories coincide which means that our reduction is coherent. For $m=1$, the transient is lost and the dynamics is approximated by the asymptotic behavior. 4) Our approach requires to know the initial condition $x^{0}$ of the original largescale system (see Proposition 1) that may be hard to achieve in practice. Therefore, we investigate the case where $x^{0}$ is subject to bounded uncertainties. The initial condition of (5) is taken to lie inside a sphere centered in $x^{0}$ and radius $\epsilon$ i. e. $\left\|x(0)-x^{0}\right\| \leq \epsilon$ with $\epsilon>0$. We see that (5) leads to

$$
x(t)=A^{t}\left(x^{0}+\left(x(0)-x^{0}\right)\right)=A^{t} x^{0}+A^{t}\left(x(0)-x^{0}\right) .
$$

By defining $\tilde{x}^{0}$ as in Proposition 1 and noting that the spectral radius of $A$ is equal to 1 in view of Assumption 1, one obtains that $\|K \tilde{x}(t)-x(t)\| \leq \varphi_{m}(t)+\epsilon$. Hence, the uncertainties will decrease the precision of the approximation but the distance between trajectories of the reduced system and the original one remains bounded.

Remark 3 At each time $t$, we may define for the community $C_{i}$ a kind of barycenter given by the $i^{\text {th }}$ component of $K^{\top} x(t)$. Then, Theorem 1 can be completed by computing the distance between the trajectory of the reduced model 
and the trajectory of the barycenters vector: $\left\|\tilde{x}(t)-K^{\top} x(t)\right\| \leq\left\|K^{\top}\right\| \| K \tilde{x}(t)-$ $x(t)\|\leq\| K \tilde{x}(t)-x(t) \| \leq \varphi_{m}(t)$ for $t \geq 0$.

\section{Dimension reduction in the stochastic matrix case}

We now consider the case where the matrix $A$ is a stochastic which is renamed $P$ in this section for the sake of clarity. A slightly different methodology is proposed. Unlike the previous case, we prove the existence of the function $\varphi_{m}(\cdot)$ but we do not give its explicit definition when $P$ is not symmetric.

This scenario is interesting since it describes the process of state synchronization which is often desired in parallel computation, negotiation, etc. The following classical conditions are assumed to hold in this section.

Assumption 2 The graph $G=(\mathcal{V}, \mathcal{E})$ is connected and $(i, i) \notin \mathcal{E}, \forall i \in 1, \ldots, n$.

That assumption implies that the matrix $P$ is irreducible and aperiodic which thus guarantees that $P$ is primitive but not necessarily symmetric as opposed to Assumption 1.

We rewrite the collective dynamics as (instead of (2))

$$
x(t+1)=P x(t), \quad \forall t \geq 0 .
$$

Let $u$ be the left Perron vector of $P$ while $\mathbf{1}_{n} \in \mathbb{R}^{n}$ (i.e. the column vector having all the entries equal 1) is the right Perron vector of $P$. Then, $\lim x(t)=x^{*} \mathbf{1}_{n}$. In this case $u_{C_{i}}=\sum_{i \in C_{i}} e_{i} \in \mathbb{R}^{n}$ which means that $\left\|u_{C_{i}}\right\|=\sqrt{\left|C_{i}\right|}$. We use the matrices $S$ and $\tilde{S}$ defined below instead of $K$ in (3)

$$
\begin{gathered}
\tilde{S}=\left(\begin{array}{c}
\frac{u_{C_{1}}^{\top}}{\left\|u_{C_{1}}\right\|^{2}} \\
\frac{u_{C_{2}}^{\top}}{\left\|u_{C_{2}}\right\|^{2}} \\
\vdots \\
\frac{u_{C_{m}}^{\top}}{\left\|u_{C_{m}}\right\|^{2}}
\end{array}\right) \in \mathbb{R}^{m \times n}, \\
S=\left(u_{C_{1}}, u_{C_{2}}, \ldots, u_{C_{m}}\right) \in \mathbb{R}^{n \times m} .
\end{gathered}
$$

We can then define a row stochastic matrix $F$ that gives the collective dynamics of communities. We note that $\tilde{S} S=I d_{m}$ and

$$
E=S \tilde{S}=\left(\begin{array}{c}
\frac{E_{1}}{\left|C_{1}\right|} \\
\frac{E_{2}}{\left|C_{2}\right|} \\
\vdots \\
\frac{E_{m}}{\left|C_{m}\right|}
\end{array}\right) \in \mathbb{R}^{n \times n}
$$


where $E_{i}$ is the matrix obtained by the juxtaposition of $\left|C_{i}\right|$ copies of $e_{C_{i}}$. The collective dynamics of the communities state is defined by

$$
\tilde{x}(t+1)=F \tilde{x}(t), \quad \forall t \geq 0
$$

where $F=\tilde{S} P S \in \mathbb{R}^{m \times m}$, therefore

$$
F=\left(f_{i j}\right)_{1 \leq i, j \leq m}, \quad f_{i j}=\frac{1}{\left|C_{i}\right|} e_{C_{i}}^{\top} P e_{C_{j}}, \quad \forall i, j .
$$

We see that an edge between $C_{i}$ and $C_{j}$ exists if and only if at least an edge between an element belonging to $C_{i}$ and one belonging to $C_{j}$ exists as for (4). In other words, $G$ is connected if and only if $\tilde{G}$ is. Moreover $P$ is irreducible if and only if $F$ is. In addition, as stated in the following lemma, the matrix $F$ is stochastic and aperiodic which implies that the Perron-Frobenius theorem applies to $F$.

Lemma $2 P$ row stochastic matrix implies $F$ row stochastic. Moreover $F$ is aperiodic if $P$ is aperiodic.

Remark $4 P$ doubly stochastic does not generally imply $F$ doubly stochastic. Indeed, consider $\tilde{S}=\left(\begin{array}{ccc}1 & 0 & 0 \\ 0 & \frac{1}{2} & \frac{1}{2}\end{array}\right), P=\left(\begin{array}{ccc}\frac{2}{3} & \frac{1}{3} & 0 \\ \frac{1}{3} & \frac{1}{3} & \frac{1}{3} \\ 0 & \frac{1}{3} & \frac{2}{3}\end{array}\right)$ doubly stochastic and $S=\left(\begin{array}{ll}1 & 0 \\ 0 & 1 \\ 0 & 1\end{array}\right)$. Then, $(1,1) F=\left(\frac{5}{6}, \frac{7}{6}\right)$, so $F$ is not column stochastic.

The result below shows us how the initial condition of the reduced model (10) needs to be selected. It is obtained by following similar lines as in the proof of Proposition 1.

Proposition 2 Consider $v$ the left Perron vector of the matrix EP. The vector $w=S^{\top} v$ is the left Perron eigenvector of $F$. If $\tilde{x}^{0}$ is chosen such that $w^{\top} \tilde{x}^{0}=u^{\top} x^{0}$ then (7) and (10) share the same consensus value meaning that $\lim _{t \rightarrow \infty}\|x(t)-S \tilde{x}(t)\|=0$.

The previous proposition ensures that Problem 1 is solved when $P$ is primitive but not necessarily symmetric. However we have not been able to depict an explicit formula for $\varphi_{m}(\cdot)$. When the graph $G$ is a connected undirected graph, then $P$ is symmetric and we can give an expression for $\varphi_{m}(\cdot)$ as in Theorem 1.

Proposition 3 Let $P$ be a symmetric stochastic matrix and $1=\lambda_{1}(P)>$ $\left|\lambda_{2}(P)\right| \geq \ldots \geq\left|\lambda_{n}(P)\right| \geq 0$ be the eigenvalues of $P$. Let also $\tilde{x}^{0}$ be chosen as in Proposition 2 and $x^{*}=\tilde{x}^{*}$ the common consensus value of the algorithms (7) and (10). A solution to Problem 1 is given by $K=S$ and

$$
\begin{aligned}
\varphi_{m}(t)= & \left|\lambda_{2}(P)\right|^{t}(\|S \tilde{x}(0)-x(0)\| \\
& \left.+t\left\|E-I d_{n}\right\|\left\|S\left(\tilde{x}(0)-x^{*} \mathbf{1}_{m}\right)\right\|\right) .
\end{aligned}
$$


Remark 5 1) Since the norm of $S$ is given by $\max _{1 \leq i \leq m}\left|C_{i}\right|$, (11) also says that large complexity reductions (i. e. $m$ very small and large communities) may lead to lower precision of the trajectory approximation close to initial conditions. Therefore, there is a trade-off between the complexity reduction and the quality of the trajectory approximation as illustrated in simulations in Section 7.

2) The $i^{\text {th }}$ component of the vector $\tilde{S} x(t) \in \mathbb{R}^{m}$ is the average of the states corresponding to the vertices belonging to the $i^{\text {th }}$ community of the network and $\|\tilde{S}\|=\frac{1}{\min _{i \in\{1, \ldots, m\}}\left|C_{i}\right|}$. Consequently, Remark 3 gives a bound on the distance between the trajectory of a community and the average of the trajectories of its elements.

\section{Numerical example}

We illustrate the theoretical results presented in Section 6. We randomly generate the initial condition with values in the interval $[0,100]$ and the interconnection graph for a network of 2000 nodes. The full model is given by (7) with $P=I d_{2000}-\alpha L$ where $L$ is the Laplacian matrix associated to the interconnection graph and $\alpha<\frac{1}{d}$ with $d$ the greatest value on the diagonal of $L$. Next, several approximations of the model are built up by following the procedure presented in Section 6. We consider the scenarios where the reduced models have 5, 10, 20, 50, 100 and 2000 nodes, respectively, in order to emphasize the trade-off between the complexity reduction and the precision of the trajectory approximation. We have observed that the simulation time decreases from more than 1 minute for 2000 nodes to about 1 second for 100 nodes. Obviously the simulation time depends on the computation capacity of our machine. Nonetheless, when the networks consists of hundred of thousands or billions of nodes the full model is not even tractable while the reduced model is. The maximum distance between the real trajectories and approximated ones over time, which is defined below, is depicted in Figure 1,

$$
\operatorname{dist}(t)=\|S \tilde{x}(t)-x(t)\|_{\infty} .
$$

One can see that this maximal distance is decreasing as a function of the size $m$ of the reduced model and for $m=n=2000$ the distance is all the time equal zero. The overall behavior of the reduced models is given in Figures 2 and 3.

\section{Conclusions and further works}

We have used a size reduction methodology to approximate the trajectories of the elements of a large scale networked system. For that purpose, we have grouped the nodes into communities. Next, we have defined the dynamics and an appropriate initial condition for the communities network. We have shown that the trajectory of a community approximate the behavior of all its elements and we have given an upper-bound on the precision of the approximation. Future 


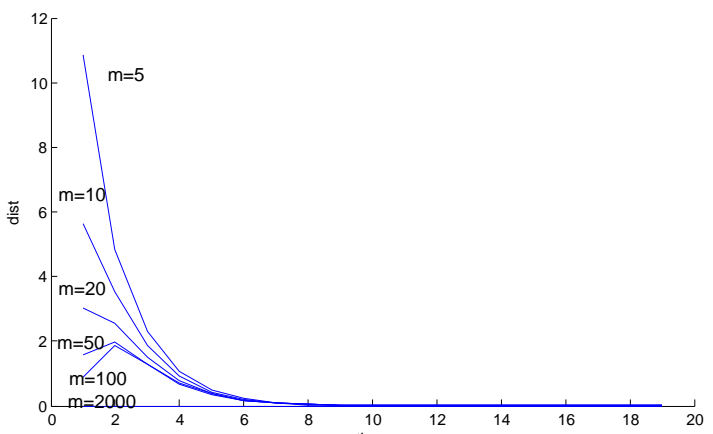

Figure 1: Evolution of the maximum distance (12) between the real trajectories and approximated ones over time for $m=5, m=10, m=20, m=50, m=100$ and $m=2000$, respectively.

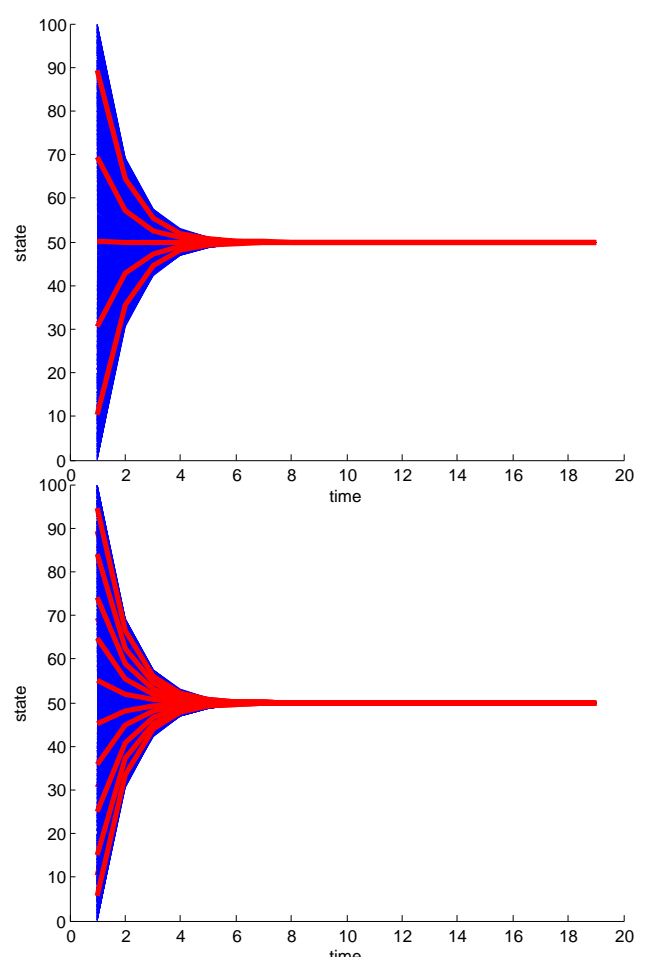

Figure 2: Blue lines represent the trajectory of the full system and the red lines the trajectory of the reduced one for $m=5$ and $m=10$, respectively.

works should consider the case of directed interaction topology described by non-symmetric matrices (as started in Section 6). An extension of these results to the case of nonlinear dynamics is also under investigation. 


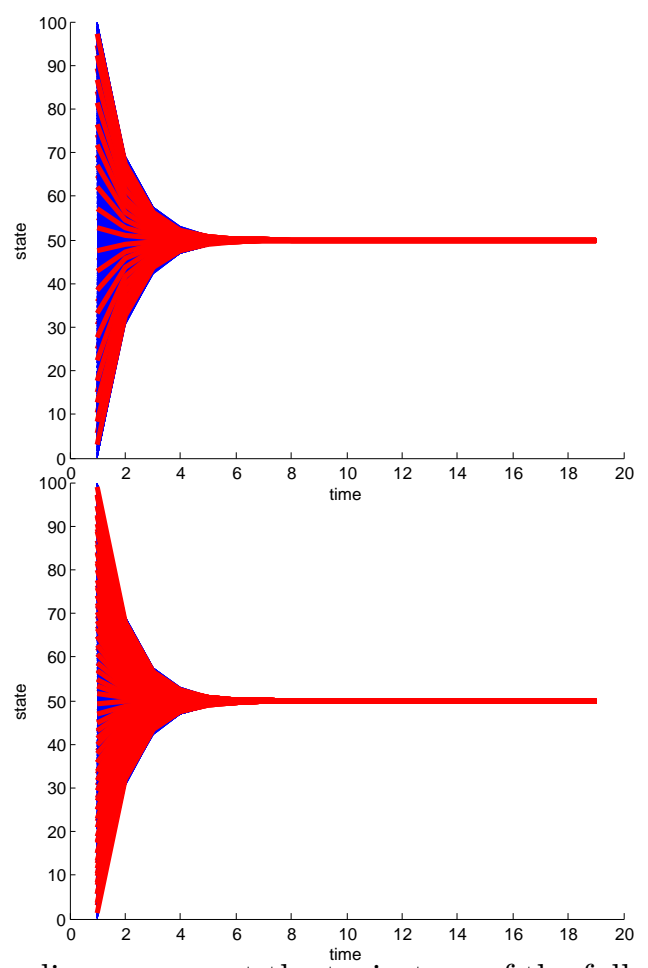

Figure 3: Blue lines represent the trajectory of the full system and the red lines the trajectory of the reduced one for $m=20$ and $m=50$, respectively.

\section{References}

[1] S. Boyd, A. Ghosh, B. Prabhakar, and D. Shah, "Randomized gossip algorithms," IEEE Trans. on Information Theory, vol. 52, no. 6, pp. 2508-2530, 2006.

[2] A. Jadbabaie, J. Lin, and A. Morse, "Coordination of groups of mobile autonomous agents using nearest neighbor rules," IEEE Transactions on Automatic Control, vol. 48, pp. 988-1001, 2003.

[3] W. Ren and R. Beard, "Consensus seeking in multiagent systems under dynamically changing interaction topologies," IEEE Transactions on Automatic Control, pp. 655-661, 2005.

[4] J. Tsitsiklis, "Problems in decentralized decision making and computation," Ph.D. dissertation, MIT, 1984.

[5] A. Antoulas, Approximation of Large-Scale Dynamical Systems. SIAM Advances in Design and Control, 2005. 
[6] A. Astolfi, "Model reduction by moment matching for linear and nonlinear systems," IEEE Trans. on Automatic Control, vol. 55, no. 10, pp. 2321$2336,2010$.

[7] I. Hanski, "Metapopulation dynamics," Nature, vol. 396, pp. 41-49, 1998.

[8] V. Colizza, R. Pastor-Satorras, and A. Vespignani, "Reaction-diffusion processes and metapopulation models in heterogeneous networks," Nature Physics, vol. 3, pp. 276-282, 2007.

[9] M. E. J. Newman and M. Girvan, "Finding and evaluating community structure in networks," Phys. Rev. E, vol. 69: 026113, 2004.

[10] R. Lambiotte, J.-C. Delvenne, and M. Barahona, "Laplacian dynamics and multiscale modular structure in networks," arXiv:0812.1770v3, 2009.

[11] V. Blondel, J.-L. Guillaume, R. Lambiotte, and E. Lefebvre, "Fast unfolding of communites in large networks," Journal of Statistical Mechanics: Theory and Experiment, vol. P10008, no. 10, 2008.

[12] I.-C. Morărescu and A. Girard, "Opinion dynamics with decaying confidence: Application to community detection in graphs," IEEE Trans. on Automatic Control, vol. 56, no. 8, pp. 1862 - 1873, 2011.

[13] D. Gfeller and P. D. L. Rios, "Spectral coarse graining and synchronization in oscillator networks," Phys. Rev. Lett., vol. 100, p. 174104, 2008.

[14] L. Moreau, "Stability of multiagent systems with time-dependent communication links," IEEE Trans. on Automatic Control, vol. 50, no. 2, pp. 169-182, 2005.

[15] P.-D. Moroşan, R. Bourdais, D. Dumur, and J. Buisson, "Building temperature regulation using a distributed model predictive control," Energy and Buildings, vol. 42, no. 9, pp. 1445-1452, 2010.

[16] C. Meyer, Matrix analysis and applied linear algebra. SIAM, 2000. 